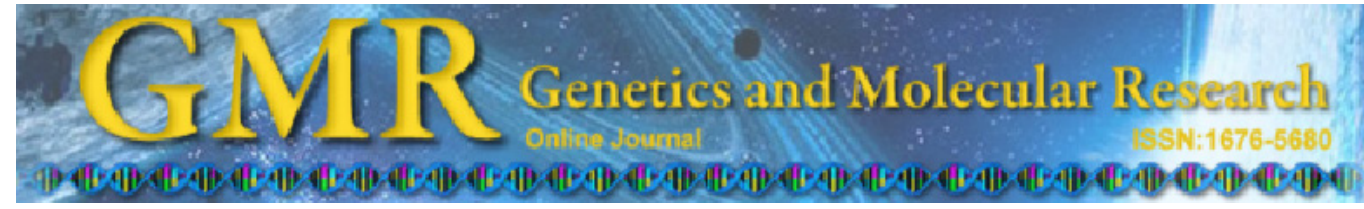

\title{
Numerical aberrations of chromosome 17 and TP53 in brain metastases derived from breast
}

\section{cancer}

\author{
D.S. Vasconcelos ${ }^{1}$, F.P.E. da Silva ${ }^{2,3}$, L.G. Quintana ${ }^{2}$, N.P. Anselmo ${ }^{4}$, \\ M.A.K. Othman ${ }^{5}$, T. Liehr ${ }^{5}$ and E.H.C. de Oliveira ${ }^{6,7}$ \\ ${ }^{1}$ Programa de Pós-Graduação em Neurociências e Biologia Celular, \\ ICB-UFPA, Belém, PA, Brasil \\ ${ }^{2}$ Programa de Pós-Graduação em Genética e Biologia Molecular, ICB-UFPA, \\ Belém, PA, Brasil \\ ${ }^{3}$ Instituto Federal de Educação, Ciência e Tecnologia do Pará, \\ Tucuruí, PA, Brasil \\ ${ }^{4}$ Laboratório de Biologia Molecular, Instituto de Ciências Biológicas, \\ Universidade Federal do Pará, Belém, PA, Brasil \\ ${ }^{5}$ Institute of Human Genetics, Jena University Hospital, \\ Friedrich Schiller University, Jena, Germany \\ ${ }^{6}$ Laboratório de Cultura de Tecidos, SAMAM, Instituto Evandro Chagas, \\ Ananindeua, PA, Brasil \\ ${ }^{7}$ Instituto de Ciências Exatas e Naturais, Faculdade de Ciências Naturais, \\ Universidade Federal do Pará, Belém, PA, Brasil \\ Corresponding author: E.H.C. de Oliveira \\ E-mail: ehco@ufpa.br/ehco@uol.com.br
}

Genet. Mol. Res. (2013) Ahead of Print

Received April 19, 2012

Accepted August 17, 2012

Published January 4, 2013

DOI http://dx.doi.org/10.4238/2013.January.4.15

ABSTRACT. Breast cancer is the second most common origin of brain metastases, after lung cancer, and represents $14-20 \%$ of all cases. Abnormalities of chromosome 17 are important molecular genetic events in human breast cancer, and several oncogenes and tumor suppressor genes are located on this chromosome. In about half of all human cancers, the tumor suppressor gene $T P 53$, located at $17 \mathrm{p} 13$, is 


\section{D.S. Vasconcelos et al.}

either lost or mutated. Loss of $\mathrm{p} 53$ protein function influences not only cell cycle checkpoint controls and apoptosis, but also the regulation of other important stages of metastatic progression, such as cell migration and tissue invasion. The aim of our study was to identify numerical aberrations of chromosome 17 and TP53 in 5 subjects with brain metastasis from breast cancer using dual-color fluorescence in situ hybridization experiments. Deletion of TP53 was the most frequent alteration observed, suggesting that if this alteration is present in the primary tumors, breast tumors with loss of TP53 copies have a poorer prognosis and a higher chance for metastasis. If this is true, the analyses of chromosome 17 and TP53 in primary breast cancer could be important for predicting its metastatic potential.

Key words: Breast cancer; TP53 gene; Chromosome 17; Metastasis; FISH

\section{INTRODUCTION}

Tumor metastasis is a multistage process in which malignant cells spread from a primary tumor to distant organs via the blood and/or lymphatic system (Fidler, 2003; Talmadge and Fidler, 2010; Saunus et al., 2011). Of the approximately 1.3 million people diagnosed with cancer in the United States each year, approximately 100,000 to 170,000 will develop brain metastases; this corresponds to an annual incidence of around 4.1 to 11.1 per 100,000 individuals (Barnholtz-Sloan et al., 2004; Cambruzzi et al., 2011). Large autopsy studies suggest that approximately 20 to $40 \%$ of all patients with metastatic cancer have brain metastases (http://www.cancer.org; Weil et al., 2005).

The most common primary metastatic sites in adults are the lung $(35 \%)$, central nervous system (CNS; 25-30\%) and breast (14-18\%; Weil et al., 2005; Cambruzzi et al., 2011; Saunus et al., 2011). This phenomenon is called organotropism, which means that the distribution of metastasis in different organs is nonrandom; i.e., the tumor cells could have specific affinity for the microenvironments of certain organs (Talmadge and Fidler, 2010; Saunus et al., 2011). Organotropism was first described by Paget (1889) after studying autopsy records of 735 women with breast cancer.

In Brazil, 52,680 new cases of breast cancer are estimated in 2012, corresponding to 52 cases in 100,000 women. In the northern region of Brazil, it is the second most frequent cancer (19/100,000 women) after cervical cancer (INCA, 2012). An immunohistochemical study of 100 Brazilian patients (Porto Alegre, RS) suggested breast cancer as the primary origin of brain metastasis in 16\% of the studied cases (Cambruzzi et al., 2011); these results confirm data ascertained in other countries, although the overall incidence may be as high as $30 \%$ according to molecular studies (Marko et al., 2012). The outcome for patients with brain metastasis derived from breast tumors is poor, and the overall survival after diagnosis varies between 2-16 months, with only $20 \%$ of patients reaching the 12-month mark (Cheng and Hung, 2007). Hence, the search for biomarkers that could help in predicting or understanding the process of metastatic events is crucial, particularly for this type of cancer.

In addition to young age and estrogen-receptor negativity, high proliferation rates, p53 
alterations, and genomic instability in the primary tumor were associated with an increased risk of CNS metastasis (Tham et al., 2006). After their spread from tumors, metastatic cells acquire the capacity to actively migrate and invade through the stroma (Muller et al., 2011). Several studies have shown that suppression of p53 can lead to more rapid migration of fibroblasts in scratch-wound assays and through 3-dimensional matrices (Guo and Zheng, 2004, Gadea et al., 2007). Other types of cells had increased growth cone motility associated with altered p53 function (Qin et al., 2009). Thus, in addition to affecting apoptosis and cellular senescence, p53 loss has recently been shown to influence cell motility, thus contributing to the invasive potential of tumors (Muller et al., 2011).

Herein, 5 breast cancer-derived brain metastases were evaluated for numerical aberrations of chromosome 17 and deletion or amplification of the tumor suppressor gene, TP53.

\section{MATERIAL AND METHODS}

Samples of breast metastatic tumors submitted for surgical resection were obtained from 5 female patients at the Ophir Loyola Hospital (Belém, PA, Brazil). The ages of the patients and histopathology of the tumors are listed in Table 1. The patients did not undergo chemotherapy or radiotherapy prior to surgery. This study was approved by the Ethics Committee of Health Sciences Center, UFPA CCS/UFPA.

\begin{tabular}{l} 
Table 1. Information about the subjects analyzed in this study. \\
\hline \begin{tabular}{llcr}
\hline Case No. & Gender & Age (year) & Histopathology \\
\hline 1 & Female & 23 & Adenocarcinoma \\
2 & Female & 54 & Adenocarcinoma \\
3 & Female & 45 & Adenocarcinoma \\
4 & Female & 46 & Adenocarcinoma \\
5 & Female & 50 & Adenocarcinoma \\
\hline
\end{tabular}
\end{tabular}

The samples were transferred to the laboratory in Roswell Park Memorial Institute medium, the material was dissociated, treated with $\mathrm{KCl}$ (incubated for $10 \mathrm{~min}$ at $37^{\circ} \mathrm{C}$ ), and fixed in methanol-acetic acid. The suspension was used for preparing slides for use in subsequent experiments. A dual-color fluorescence in situ hybridization assay was performed using directly labeled fluorescent probes (SpectrumRed and SpectrumGreen) for chromosome 17. A centromere-specific probe (cep) and a locus-specific probe for the gene TP53 on 17p13.1 were applied following manufacturer protocols (Abbott, Abbott Park, IL, USA; Vysis, Des Plains, IL, USA). After stringency washes, a drop of antifade solution (Vectashield, Vector Laboratories, Burlingame, CA, USA) containing 4',6-diamidino-2-phenylindole for counterstaining was added and each slide was covered with a 32 x 22 coverslip.

The slides were analyzed using a Zeiss Axio Imager epifluorescent microscope (Zeiss, Jena, Germany) at a magnification of $600 \mathrm{X}$. Images were captured and analyzed using the Axiophot 4.1 software. For each subject, 160 to 200 interphase nuclei were evaluated. Counting was performed using criteria proposed by Hopman et al. (1994). We considered the occurrence of monosomy (1 signal for TP53 and centromere 17), normal (2 signals for each probe) and polysomy (more than 2 signals for each probe). For the TP53 gene, we considered the occurrence of deletions (less than 2 signals), normal (2 signals) or amplification (more than 2 signals). 


\section{D.S. Vasconcelos et al.}

For statistical analysis of the results, we used the non-parametric Mann-Whitney Utest, with $\mathrm{P}<0.05$ considered significant (Espinosa et al., 2006).

\section{RESULTS}

All 5 subjects presented to a certain extent with numerical alterations of chromosome 17 as well as copy number alterations (deletion or amplification) of TP53 in the studied metastases (Figure 1). Three of the 5 cases showed 1 or no signal for TP53 in 19.4 to $49 \%$ of cells, respectively, while 2 cep 17 signals were present in each sample. The other 2 cases had 2 signals for each probe in 26 and 50\% of cells. For the latter 2 samples, a deletion of TP53 was present in 33.7 and $34 \%$ of cells, respectively. In all 5 samples, gain of chromosome 17 was rare, varying from 0.5 to $8.4 \%$ of the analyzed cells. Statistical analyses are shown in Table 2 .

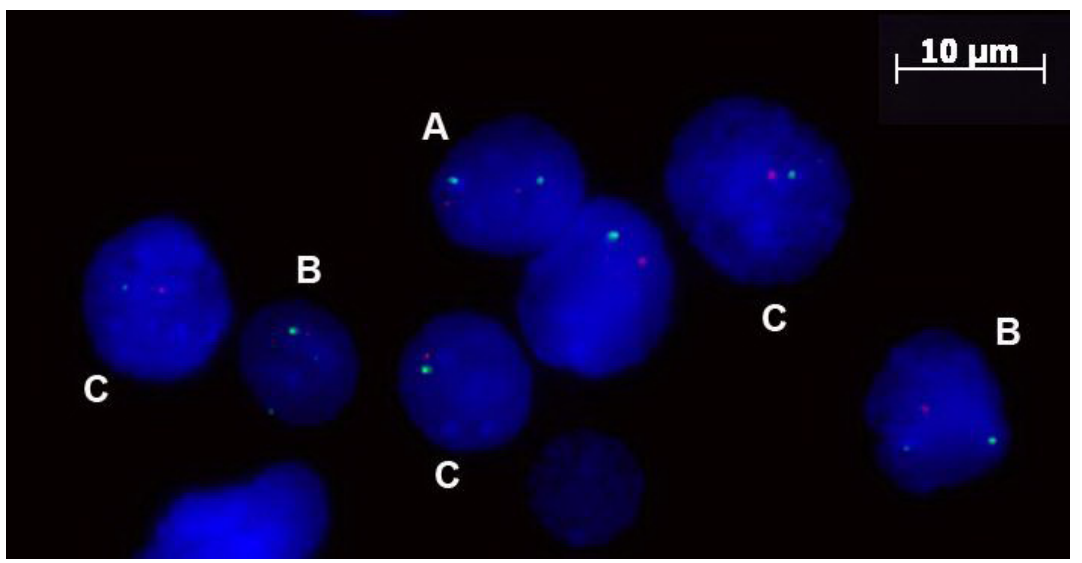

Figure 1. Representative dual color fluorescence in situ hybridization experiments with centromeric probe of chromosome 17 (green) and locus-specific probe for TP53 (17p13.1; red) in interphase nuclei: (A) nucleus, with two copies of chromosome 17 and TP53; (B) nuclei with two copies of chromosome 17 and deletion of one TP53; (C) nuclei with deletion of one chromosome 17, as one green and one red signal are absent.

Table 2. Main cytogenetics findings and statistical analyses.

\begin{tabular}{lccccr}
\hline Case No. & No alteration (\%) & Trisomy 17 (\%) & Monosomy 17 (\%) & Deletion of p53 (\%) & Number of analyzed cells \\
\hline 1 & 33.7 & 3.0 & 15.6 & 38.7 & 160 \\
2 & 34.0 & 2.0 & 10.0 & 49.0 & 200 \\
3 & 50.0 & 0.5 & 9.5 & 34.0 & 200 \\
4 & 26.3 & 8.4 & 6.8 & 18.9 & 190 \\
5 & 16.8 & 8.4 & 6.3 & 19.4 & 190 \\
\hline
\end{tabular}

\section{DISCUSSION}

Breast cancer is a heterogeneous disease with respect to molecular features, which is perhaps best exemplified by the molecular subgroups identifiable by gene expression profiling including basal-like, luminal $\mathrm{A}$ (hormone receptor positive), luminal $\mathrm{B}$, and human epidermal growth factor receptor 2 (HER2) amplified/over-expressed (HER2+) subtypes (Saunus et al., 
Aberrations of chromosome 17 and TP53 in brain metastasis

2011). It has been demonstrated that primary breast cancer with a basal-like immunophenotype has the propensity to metastasize to the brain (Espinosa et al., 2006). Indeed, breast cancer is the second most common source of brain metastases after lung cancer, representing $14-20 \%$ of all cases (Fulford et al., 2007; Cambruzzi et al., 2011). Although the incidence of breast metastasis in the brain is high, and metastatic transition involves the occurrence of many chromosomal abnormalities (Lohr et al., 2001), little is known about the behavior of chromosomes in metastases.

The molecular basis for breast cancer metastasis to the brain is largely unknown (Gagos and Irminger-Finger, 2005). Some studies have shown that breast cancer metastasis to the brain involves mediators of extravasation through non-fenestrated capillaries, complemented by specific enhancers of blood-brain barrier crossing and brain colonization, involving cyclooxygenase 2 (COX2; also known as PTGS2), the epidermal growth factor receptor ligand HBEGF, and the $\alpha 2,6$-sialyltransferase ST6GALNAC5 as mediators of cancer cell passage through the blood-brain barrier (Chiang and Massagué, 2008). Moreover, the importance of mutations in $\mathrm{p} 53$ protein has also been highlighted due to the influence of these mutations in tumor cell migration and invasion (Muller et al., 2011). Studies involving the analyses of modifications in the copy number of the TP53 gene, located at $17 \mathrm{p} 13.1$, have also demonstrated the occurrence of abnormalities in the number of copies of chromosome 17.

Two different studies focusing on breast metastases in lymph nodes have found loss of chromosome 17 to be the most common chromosomal abnormality (Pandis et al., 1998; Bos et al., 2009). Abnormalities of chromosome 17 are important molecular genetic events in human breast cancer as a whole, which include several widely studied oncogenes and tumor suppressor genes (Tsuda et al., 1998). Hence, the deletion of tumor suppressor genes or the amplification of oncogenes is an important step that can be crucial in the process of the development and progression of the primary tumor and its metastases. High rates of abnormalities involving chromosome 17 have been reported in some human cancers, including breast (Zhang and Yu, 2011), colon (Hopman et al., 1994), and bladder carcinoma (Rosenberg et al., 1994). This chromosome has a high content of guanine and cytosine, regions rich in important genes, a high number of short interspersed elements and a lack of long interspersed elements (Fadl-Elmula, 2005). Besides TP53, several oncogenes, such as HER2, topoisomerase (DNA) II alpha and microtubule-associated protein tau; other tumor suppressor genes (breast cancer 1 -BRCA1- and hypermethylated in cancer 1-HIC-1) or DNA double-strand break repair gene (RDM1) are located in chromosome 17 (Meszaros et al., 2010). Thus, abnormalities of chromosome 17 are important molecular genetic players in tumorigenesis, particularly in breast cancer (Reinholz et al., 2009).

Deletion of TP53 was the most frequent alteration observed in our samples (Figure 1). If we consider that this alteration could be present in the primary tumors, it could be argued that primary tumors with loss of TP53 gene copies had a poorer prognosis and a higher probability of metastasis. If this is true, analyses of chromosome 17 and TP53 in primary breast cancer could be valuable in predicting its metastatic potential.

\section{ACKNOWLEDGMENTS}

The authors would like to thank the Ophir Loyola Hospital (Belém-PA-Brazil), CNPq and Deutscher Akademischer Austausch Dients (DAAD). 
D.S. Vasconcelos et al.

\section{REFERENCES}

Barnholtz-Sloan JS, Sloan AE, Davis FG, Vigneau FD, et al. (2004). Incidence proportions of brain metastases in patients diagnosed (1973 to 2001) in the Metropolitan Detroit Cancer Surveillance System. J. Clin. Oncol. 22: 2865-2872.

Bos PD, Zhang XH, Nadal C, Shu W, et al. (2009). Genes that mediate breast cancer metastasis to the brain. Nature 459: 1005-1009.

Cambruzzi E, Pêgas KL and Ferrari MB (2011). Avaliação imuno-histoquímica de 100 casos de metástases encefálicas e correlação com o sítio primário do tumor. J. Bras. Patol. Med. Lab. 47: 57-64.

Cheng X and Hung MC (2007). Breast cancer brain metastases. Cancer Metastasis Rev. 26: 635-643.

Chiang AC and Massagué J (2008). Molecular basis of metastasis. N. Engl. J. Med. 359: 2814-2823.

Espinosa AB, Tabernero MD, Maillo A, Sayagues JM, et al. (2006). The cytogenetic relationship between primary and recurrent meningiomas points to the need for new treatment strategies in cases at high risk of relapse. Clin. Cancer Res. 12: 772-780.

Fadl-Elmula I (2005). Chromosomal changes in uroepithelial carcinomas. Cell Chromosome 4: 1.

Fidler IJ (2003). The pathogenesis of cancer metastasis: the 'seed and soil' hypothesis revisited. Nat. Rev. Cancer 3: 453 458.

Fulford LG, Reis-Filho JS, Ryder K, Jones C, et al. (2007). Basal-like grade III invasive ductal carcinoma of the breast: patterns of metastasis and long-term survival. Breast Cancer Res. 9: R4.

Gadea GMT, Angulle C and Roux P (2007). Loss of p53 promotes RhoA-ROCK-dependent cell migration and invasion in 3D matrices. J. Cell Biol. 178: 23-30.

Gagos S and Irminger-Finger I (2005). Chromosome instability in neoplasia: chaotic roots to continuous growth. Int. $J$. Biochem. Cell Biol. 37: 1014-1033.

Guo F and Zheng Y (2004). Rho family GTPases cooperate with p53 deletion to promote primary mouse embryonic fibroblast cell invasion. Oncogene 23: 5577-5585.

Hopman AH, Voorter CE and Ramaekers FC (1994). Detection of genomic changes in cancer by in situ hybridization. Mol. Biol. Rep. 19: 31-44.

INCA (2012). Instituto Nacional do Câncer. Available at [http://www.inca.gov.br]. Accessed January 20, 2012.

Lohr F, Pirzkall A, Hof H, Fleckenstein K, et al. (2001). Adjuvant treatment of brain metastases. Semin. Surg. Oncol. 20: $50-56$.

Marko NF, Xu Z, Gao T, Kattan MW, et al. (2012). Predicting survival in women with breast cancer and brain metastasis: A nomogram outperforms current survival prediction models. Cancer 118: 3749-3757.

Meszaros N, Belengeanu D, Stoicanescu D, Andreescu N, et al. (2010). Analysis of numerical aberrations of chromosome 17 and tp53 gene deletion/amplification in human oral squamous cell carcinoma using dual-color fluorescence in situ hybridization. Anal. Univ. Oradea - Fascicula Biologie XVII/1: 142-146.

Muller PA, Vousden KH and Norman JC (2011). p53 and its mutants in tumor cell migration and invasion. J. Cell Biol. 192: 209-218.

Paget S (1889). The distribution of secondary growths in cancer of the breast. Lancet 133: 571-573.

Pandis N, Teixeira MR, Adeyinka A, Rizou H, et al. (1998). Cytogenetic comparison of primary tumors and lymph node metastases in breast cancer patients. Genes Chromosomes Cancer 22: 122-129.

Qin Q, Baudry M, Liao G, Noniyev A, et al. (2009). A novel function for p53: regulation of growth cone motility through interaction with Rho kinase. J. Neurosci. 29: 5183-5192.

Reinholz MM, Bruzek AK, Visscher DW, Lingle WL, et al. (2009). Breast cancer and aneusomy 17: implications for carcinogenesis and therapeutic response. Lancet Oncol. 10: 267-277.

Rosenberg C, Andersen TI, Nesland JM, Lier ME, et al. (1994). Genetic alterations of chromosome 17 in human breast carcinoma studied by fluorescence in situ hybridization and molecular DNA techniques. Cancer Genet. Cytogenet. 75: $1-5$.

Saunus JM, Momeny M, Simpson PT and Lakhani SR (2011). Molecular aspects of breast cancer metastasis to the brain. Genet. Res. Int. 2011: 9.

Talmadge JE and Fidler IJ (2010). AACR centennial series: The biology of cancer metastasis: Historical perspective. Cancer Res. 70: 5649-5669.

Tham YL, Sexton K, Kramer R, Hilsenbeck S, et al. (2006). Primary breast cancer phenotypes associated with propensity for central nervous system metastases. Cancer 107: 696-704.

Tsuda H, Sakamaki C, Tsugane S, Fukutomi T, et al. (1998). A prospective study of the significance of gene and chromosome alterations as prognostic indicators of breast cancer patients with lymph node metastases. Breast Cancer Res. Treat. 48: 21-32. 
Aberrations of chromosome 17 and TP53 in brain metastasis

Weil RJ, Palmieri DC, Bronder JL, Stark AM, et al. (2005). Breast cancer metastasis to the central nervous system. Am. J. Pathol. 167: 913-920.

Zhang W and Yu Y (2011). The important molecular markers on chromosome 17 and their clinical impact in breast cancer. Int. J. Mol. Sci. 12: 5672-5683. 